\title{
Ovarian function after bone marrow transplantation performed before menarche
} M Matsumoto, O Shinohara, H Ishiguro, T Shimizu, K Hattori, M Ichikawa, H Yabe,
C Kubota, M Yabe, S Kato
Department of

Pediatrics, Tokai

University School of

Medicine, Bohseidai,

Isehara-shi,

Kanagawa-ken

259-1193, Japan

M Matsumoto

O Shinohara

$\mathrm{H}$ Ishiguro

T Shimizu

K Hattori

M Ichikawa

$\mathrm{H}$ Yabe

C Kubota

M Yabe

Research Center for Genetic Engineering and Cell

Transplantation, Tokai

University School of

Medicine

S Kato

Correspondence to:

Dr Shinohara.

email: oshinoha@

is.icc.u-tokai.ac.jp

Accepted 26 January 1999

\begin{abstract}
Aim-To examine the long term effect of bone marrow transplantation (BMT) on ovarian function in girls.

Methods-Eighteen girls who underwent BMT before menarche, had been disease free for more than six years, and were over 14 years of age at the time of study were investigated. The preparative regimen consisted of irradiation and chemotherapy. The occurrence of menarche and changes in basal serum follicle stimulating hormone (FSH) concentrations were studied.

Results-Twelve patients achieved menarche at a median age of 12.8 years. Age at transplant was significantly younger in patients who achieved menarche than in those who did not (mean (SD), $7.2(0.5) v$ 11.1 (1.7) years). Basal FSH began to rise to menopausal concentrations after 10 years of age, and the girls who did not experience menarche had a sustained rise in FSH concentrations. Among those with raised FSH concentrations, five girls experienced menarche while serum FSH values were decreasing and four achieved menarche while FSH remained raised.

Conclusions-The high incidence of menarche suggests a favourable outcome of ovarian function in girls who undergo BMT at a young age.

(Arch Dis Child 1999;80:452-454)
\end{abstract}

Keywords: bone marrow transplantation;

gonadotropins; gonadal failure; menstruation

Bone marrow transplantation (BMT) has become an established treatment for haematological malignancies, such as leukaemia and non-malignant haematopoietic diseases including severe aplastic anaemia. Because the number of survivors of BMT is increasing, the assessment of long term side effects has become an important issue..$^{1-5}$ From an endocrine point of view, primary gonadal failure as a result of either high dose alkylating agents or irradiation given as a preparative regimen is one of the serious late side effects. Previous reports showed the long term nature of gonadal impairment, which tended to be more severe among female patients than among men. Furthermore, girls/women who received BMT after menarche suffered more prolonged and severe gonadal impairment than those transplanted before menarche. ${ }^{25}$ Recently, Sarafoglou et al have shown that at least $50 \%$ of female patients who underwent BMT before puberty achieved menarche despite raised plasma gonadotropin concentrations. ${ }^{6}$ Attaining normal gonadal function is an important issue for long term survivors when considering their quality of life. Our study describes the occurrence of menarche and the changes in gonadotropin secretion among patients who were premenarcheal at the time of BMT.

\section{Patients and methods}

We reviewed the records of the girls who: (1) underwent BMT before menarche, (2) had been surviving free from the original disease for more than six years, and (3) were over 14 years of age at the time of our study. Between March 1982 and May 1991, 100 patients (57 boys and 43 girls) underwent allogenic BMT. Of the 43 female patients, 16 died and we reviewed the records of the remaining 27 disease-free survivors. Twenty four patients were premenarcheal at the time of BMT. Three were excluded from the analysis because of a lack of sufficient data, and an additional three patients who were under 14 years of age at the time of our study were left out. The remaining 18 patients fulfilled the criteria and were included in our study. All patients except for two (patients 17 and 18) were prepubertal at the time of BMT.

Fourteen had haematological malignancies: 12 with leukaemia and two with nonHodgkin's lymphoma. Four girls had nonmalignant haematological disorders: four with aplastic anaemia, including one patient with Fanconi's anaemia. The age at BMT ranged from 4.5 to 15.3 years (mean (SD), 8.5 (3.2) years), and the age at study ranged from 14.4 to 24.6 years (mean (SD), 18.4 (3.2) years). The follow up duration ranged from 6.9 to 12.9 years (mean (SD), 9.9 (1.8) years). The preparative regimen consisted of irradiation and chemotherapy. Fractionated total body irradiation (TBI) of 8-12 Gy was given to patients with malignant diseases, and those with non-malignant disorders received 6-8 Gy of either TBI or thoraco-abdominal irradiation (TAI). Chemotherapy consisted of cyclophosphamide $(50-60 \mathrm{mg} / \mathrm{kg}$ once daily intravenously for two to four days; total dose, $120-200 \mathrm{mg} / \mathrm{kg})$, cytosine arabinoside $\left(3 \mathrm{~g} / \mathrm{m}^{2}\right.$ twice daily intravenously for five days; total dose $30 \mathrm{~g} / \mathrm{m}^{2}$ ), etoposide (50-60 $\mathrm{mg} / \mathrm{kg}$ once daily intravenously for one day), and antilymphocyte globulin, either alone or in combination. Prevention of graft versus host disease (GVHD) was attempted by the administration of cyclosporin A (3 mg/kg/day intravenously) and methotrexate $(15 \mathrm{mg} / \mathrm{kg} /$ day intravenously initially and $10 \mathrm{mg} / \mathrm{kg} /$ day for three times thereafter). 
Table 1 Profile of the patients

\begin{tabular}{|c|c|c|c|c|c|c|c|c|}
\hline Patient & Diagnosis & $\begin{array}{l}\text { Age at } B M T \\
\text { (years) }\end{array}$ & $\begin{array}{l}\text { Age at menarche } \\
\text { (years) }\end{array}$ & $\begin{array}{l}\text { Age at study } \\
\text { (years) }\end{array}$ & $T B I(G y)$ & $T A I(G y)$ & Chemotherapy & $c G V H D$ \\
\hline 1 & AA & 4.5 & 12.8 & 15.9 & - & 8 & $\mathrm{CY}$ & - \\
\hline 2 & AA & 4.9 & 12.8 & 15.0 & - & 8 & $\mathrm{CY}$ & - \\
\hline 3 & CML & 5.3 & 11.0 & 14.4 & 12 & - & Ara-C, VP16 & - \\
\hline 4 & ALL & 5.7 & 11.4 & 16.2 & 12 & - & VP16 & - \\
\hline 5 & CML & 6.4 & 11.0 & 19.3 & 12 & - & CY, Ara-C & - \\
\hline 6 & ALL & 7.3 & 13.3 & 16.0 & 12 & - & Ara-C, VP16 & - \\
\hline 7 & NHL & 7.5 & 14.0 & 15.8 & 12 & - & CY, VP16 & - \\
\hline 8 & AML & 7.9 & 10.8 & 19.0 & 12 & - & CY, Ara-C & - \\
\hline 9 & ALL & 8.2 & 13.3 & 15.1 & 12 & - & Ara-C, VP16 & - \\
\hline 10 & ALL & 9.0 & 13.3 & 16.0 & 12 & - & Ara-C, VP16 & - \\
\hline 11 & AA & 9.7 & 12.3 & 22.6 & 8 & - & CY & + \\
\hline 12 & NHL & 10.0 & 13.8 & 18.0 & 12 & - & Ara-C, VP16 & - \\
\hline 13 & ALL & 4.6 & - & 16.0 & 12 & - & CY, Ara-C & - \\
\hline 14 & Fanconi & 8.4 & - & 19.9 & - & 6 & CY, ALG & + \\
\hline 15 & AML & 11.1 & - & 22.6 & 12 & - & CY, Ara-C & + \\
\hline 16 & AML & 12.3 & - & 21.3 & 12 & - & Ara-C, VP16 & - \\
\hline 17 & AML & 15.0 & - & 24.6 & 12 & - & Ara-C, VP16 & - \\
\hline 18 & AML & 15.3 & - & 23.4 & 12 & - & Ara-C, VP16 & - \\
\hline
\end{tabular}

AA, aplastic anaemia; CML, chronic myelogenous leukaemia; ALL, acute lymphoblastic leukaemia; NHL, non-Hodgkin's lymphoma; AML, acute myeloid leukaemia; Fanconi, Fanconi's anaemia; BMT, bone marrow transplantation; TBI, total body irradiation; TAI, thoraco-abdominal irradiation; CY, cyclophosphamide; Ara-C, cytosine arabinoside; VP16, etoposide; ALG, antilymphocyte globulin; cGVHD, chronic graft versus host disease.

We recorded the time of the occurrence of menarche and we assessed gonadotropin secretion by the gonadotrophin releasing hormone (GnRH) loading test $\left(100 \mu \mathrm{g} / \mathrm{m}^{2}\right.$; maximum $100 \mu \mathrm{g})$ performed on repeated occasions. Because basal luteinising hormone $(\mathrm{LH})$ values, as well as peak follicle stimulating hormone $(\mathrm{FSH})$ values, correlated closely with basal FSH concentrations, we only describe changes in basal FSH concentrations in our report. We defined serum FSH concentrations $>20 \mathrm{IU} / 1$ as raised, indicating the presence of primary gonadal failure. We performed statistical analysis with the Mann-Whitney test. FSH was measured by enzyme immunoassay.

\section{Results}

Twelve patients entered puberty spontaneously and achieved menarche at median age of 12.8 years (range, 10.8 to 14.0 years) (table 1 ). These girls received marrow grafting at 4.5 to 10.0 years of age. Of the 14 girls who had

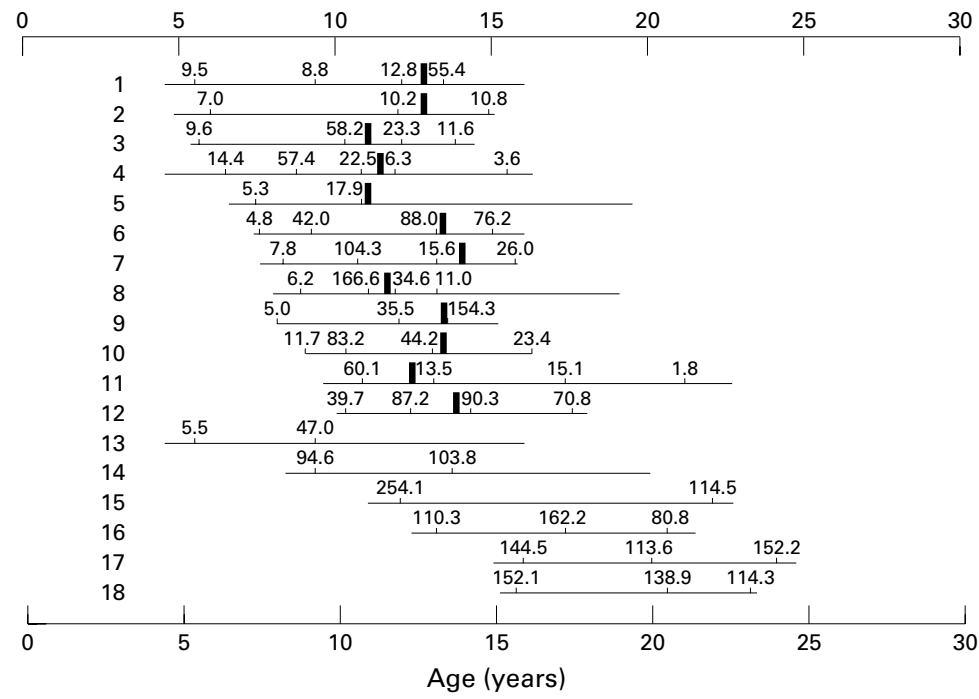

Figure 1 Changes in basal follicle stimulating hormone (FSH) concentrations in relation to the time of appearance of menarche after bone marrow transplantation (BMT). Numbers on the left side denote patient number. The beginning and end of each horizontal line denote the age at BMT and the age at the time of study, respectively. Thick vertical lines show the time of menarche. Numbers above each horizontal lines denote basal serum FSH concentrations (IU/l). received grafting before 10 years of age, 12 achieved menarche, whereas none of the four patients who underwent BMT after 11 years of age experienced menarche. The patients who achieved menarche were significantly younger compared with those who did not (mean (SD), $7.2(0.5) v 11.1(1.7)$ years; $\mathrm{p}=0.04)$.

Menarche occurred in nine of 14 and three of four girls with malignant and non-malignant disease, respectively. Ten of 15 patients who had received fractionated TBI (8-12 Gy) before BMT underwent menarche. Among these children, six of nine and four of six patients without and with cyclophosphamide administration, respectively, experienced menarche. Two of three patients who had received TAI and cyclophosphamide achieved menarche. Of the three patients who developed chronic GVHD according to the criteria of Shulman et $a l^{7}$ one experienced menarche (table 1).

Serial examination of gonadotropin concentrations revealed a tendency of basal FSH to rise to menopausal values in many patients after 10 years of age (fig 1 ). All patients except one (patient 2) experienced raised basal FSH concentrations indicating the presence of ovarian failure at some time during the follow up period. Although many girls had sustained rises of gonadotropin values, raised FSH concentrations returned to normal in some patients (patients 3, 4, 7, 8, and 11). In general, the patients who did not experience menarche (patients 13-18) had continuously raised basal FSH concentrations compared with those who did (patients 1-12).

Close observation of the changes in basal FSH values around the time of menarche showed diverse patterns. Two patients (patients 1 and 2) continued to have normal FSH concentrations until menarche. One of these (patient 1) had raised FSH concentrations six months after menarche. Five girls (patients 3, $4,7,8$, and 11) achieved menarche while their serum FSH concentrations were decreasing towards normal. One patient (patient 8) had greatly raised FSH concentrations (166.6 IU/1) just before menarche at the age of 10.8 years. 
Four girls (patients 6, 9, 10, and 12) underwent menarche despite continuously raised $\mathrm{FSH}$ concentrations. No patients showed precocious development of puberty.

\section{Discussion}

In our study, we found a high incidence of menarche in patients who underwent BMT. Furthermore, serum FSH values remained raised despite the development of menses in five patients. These findings agree with the recent report by Sarafoglou et al. ${ }^{6}$

Gonadal failure is one of the serious endocrine consequences of BMT. It has been repeatedly stated that the ovary is susceptible to long lasting damage induced by irradiation or high dose chemotherapy given as preparative treatment. ${ }^{125}$ Recovery of ovarian function has been described recently, ${ }^{68}$ and successful pregnancy was also reported. ${ }^{9}{ }^{10}$ However, the relation between the occurrence of menstruation and changes in gonadotropin concentrations has not been described in detail until recently. ${ }^{6}$ Sex, age at transplantation, or type of irradiation (single fraction or fractionated) are important determinants of the gonadal outcome. ${ }^{34}$

Basal serum FSH values remained low until around 10 years of age, and gradually rose to hypogonadal values thereafter, resembling the change found in Turner's syndrome. ${ }^{11}$ The occurrence of menarche under the condition where basal gonadotropin concentrations remain raised has been described recently. ${ }^{6}$ The ovary of young patients is reported to be more resistant to irradiation, so that the remaining undamaged follicles might later respond to raised gonadotropins. ${ }^{6}$ We were surprised to find a high rate of occurrence (nine of 14) of menarche among patients with malignant diseases-who had received 12 Gy of TBI. Comparison of the effect of the mode of irradiation (TBI $v$ TAI) on gonadal function was not feasible because of the small number of patients receiving TAI.

Age at BMT has been reported to be the main determinant of subsequent gonadal function. ${ }^{2568}$ Our study showed that there was a high incidence of menstruation among those who had undergone transplantation before 10 years of age, whereas none of the patients who underwent BMT after 10 years of age achieved menarche. Thus, it can be assumed that the incidence of ovarian dysfunction is low if patients undergo marrow grafting at a younger age. Even if basal FSH concentrations exceeded $100 \mathrm{IU} / 1$, recovery of ovarian function is possible, as was seen in patients 7 and 8 .

Both alkylating agents and irradiation are known to damage gonadal function. Our data showed that the rate of menarche did not decrease when cyclophosphamide was added to TBI as a preparative treatment. This implies that TBI is mainly responsible for ovarian dysfunction and that high dose cyclophosphamide had no additional deleterious effect on ovarian function. Most of the patients who had not received cyclophosphamide were given etoposide. The effect of etoposide on gonadal function has not been described.

In summary, a significantly high incidence of menarche was seen, particularly in girls who received BMT before 10 years of age, despite the presence of a continuous rise in basal serum FSH. These data suggest a favourable outcome of ovarian function in patients who undergo BMT before menarche. However, it is too optimistic to predict that those who achieved menarche will become fertile because most of these patients still have raised basal FSH concentrations to some degree. A definite conclusion concerning the recovery from ovarian dysfunction cannot be drawn until all of the patients in our study reach postpubertal age. Careful follow up study is necessary to determine the outcome of gonadal function and the reproductive potential of bone marrow recipients.

This work was supported in part by a grant from the Scientific Frontier Program of the Ministry of Education, Science and Sports, and by the Grant-in-Aid for Cancer Research (10-1) from the Ministry of Health and Welfare.

1 Sklar CA, Kim TH, Williamson JF, Ramsay NK. Ovarian function after successful bone marrow transplantation in
postmenarcheal females. Med Pediatr Oncol 1983;11:361-4.

2 Sanders JE, Buckner CD, Amos D, et al. Ovarian function following marrow transplantation for aplastic anemia or following marrow transplantation for

3 Ogilvy-Stuart AL, Clark DJ, Wallace WH, et al. Endocrine deficit after fractionated total body irradiation. Arch Dis Child 1992;67:1107-10.

4 Thomas BC, Stanhope R, Plowman PN, Leiper AD. Endocrine function following single fraction and fractionated total body irradiation for bone marrow transplantation in childhood. Acta Endocrinol (Copenh) 1993;128:508-12.

5 Chatterjee R, Goldstone AH. Gonadal damage and effects on fertility in adult patients with haematological malignancy undergoing stem cell transplantation. Bone Marrow Transplant 1996; 17:5-11.

6 Sarafoglou K, Boulad F, Gillio A, Sklar C. Gonadal function after bone marrow transplantation for acute leukemia during childhood. F Pediatr 1997;130:210-16.

7 Shulman HM, Sullivan KM, Weiden PL, et al. Chronic graft-versus-host syndrome in man. A long-term clinicopathologic study of 20 Seattle patients. Am $\mathcal{F}$ Med 1980;69: 204-17.

8 Spinelli S, Chiodi S, Bacigalupo A, et al. Ovarian recovery after total body irradiation and allogeneic bone marrow transplantation: long-term follow up of 79 females. Bone Marrow Transplant 1994;14:373-80.

9 Milliken S, Powles R, Parikh PI, et al. Successful pregnancy following bone marrow transplantation for leukaemia. Bone Marrow Transplant 1990;5:135-7.

10 Sanders JE, Hawley J, Levy W, et al. Pregnancies following high-dose cyclophosphamide with or without high-dose high-dose cyclophosphamide with or without high-dose busulfan or total-body irradiation and
plantation. Blood 1996;87:3045-52.

11 Conte FA, Grumbach MM, Kaplan SL. A diphasic pattern of gonadotropin secretions in patients with the syndrome of gonadal dysgenesis. F Clin Endocrinol Metab 1975;40:670- 